\title{
Effect of Resveratrol Supplementation on the Performance of Dogs in an Eight-Arm Radial Maze
}

\author{
Michelle M. Story ${ }^{1}$, Jacquie S. Rand ${ }^{*}, 1$, Melissa Shyan-Norwalt ${ }^{2}$, Rita Mesch ${ }^{1}$, John M. Morton ${ }^{1,4}$, \\ Elizabeth A. Flickinger ${ }^{3}$
}

${ }^{1}$ Centre for Companion Animal Health, School of Veterinary Science, The University of Queensland, St Lucia, Queensland, 4072, Australia

${ }^{2}$ Companion Animal Problem Solvers, Inc, 11571 West US Highway 40, Cambridge City, Indiana, 47327, USA

${ }^{3}$ P\&G Pet Care, Dayton, Ohio, USA

${ }^{4}$ PO Box 2277, Geelong, Victoria, 3220, Australia

\begin{abstract}
Resveratrol supplements are marketed on the premise they enhance cognitive function. We explored effects of resveratrol supplementation on cognitive function, in the absence of pathology, by assessing effects of resveratrol on the performance of young, healthy, recently gonadectomised dogs in an eight-arm radial maze. Effects of gonadectomy on cognition were also assessed. Performances of untreated intact dogs, resveratrol-treated gonadectomised dogs (60mg/day of resveratrol orally) and placebo-treated gonadectomised dogs were compared in three phases. Dogs received the placebo or resveratrol for two to four weeks before testing began. In phase one, untreated intact $(n=16)$, resveratrol-treated gonadectomised $(n=6)$ and placebo-treated gonadectomised dogs $(n=5)$ were allowed to choose all maze arms they entered. In phase two, untreated intact $(n=15)$, resveratrol-treated gonadectomised $(n=6)$ and placebo-treated gonadectomised dogs $(n=5)$ entered four predetermined arms and then made four free choices from all eight arms. In phase three, a subset of dogs previously tested as untreated intact dogs in phases one and two were tested. In phase three, placebotreated gonadectomised $(n=6)$ and resveratrol-treated gonadectomised dogs $(n=6)$ entered seven predetermined arms and then had free choice between a previously opened arm and the unopened arm. Performance in the maze did not differ significantly between gonadectomised dogs receiving the placebo and gonadectomised dogs receiving resveratrol or between untreated intact dogs and gonadectomised dogs receiving the placebo. We conclude that resveratrol treatment does not improve (and gonadectomy does not impair) the aspects of cognitive function that were tested by the radial arm maze trials in young, healthy dogs receiving extensive handling and environmental/behavioural enrichment.
\end{abstract}

Keywords: Resveratrol, gonadectomy, radial arm maze, dogs, memory, cognition.

\section{INTRODUCTION}

Resveratrol, a plant-derived phytoestrogen, may minimise the deterioration in cognitive function associated with physical and chemical damage to the brain [1-7]. In rats and mice, resveratrol has been found to minimise the adverse brain and cognitive changes induced by neurologically damaging events such as streptozotocin-induced diabetes [1]; percussive cerebral trauma [2]; focal cerebral ischemia (stroke) [3]; oxidative stress caused by intracerebroventricular injection of streptozotocin [4] or cochinine [5]; and neurotoxic substances used to mimic Huntington's disease [6] and Parkinson's disease [7]. Resveratrol has been shown to decrease lipid peroxidation and up-regulate antioxidant enzymes in the brains of healthy male rats [8]. This indicates that resveratrol may protect against oxidative damage in the brain.

\footnotetext{
*Address correspondence to this author at the Centre for Companion Animal Health, School of Veterinary Science, The University of Queensland, St Lucia, Queensland, 4072, Australia; Tel: +61 73365 2122;

Fax: +61 73346 9822; E-mails: j.rand@uq.edu.au
}

Resveratrol appears to provide some protection against age-related deterioration in cognitive function. In a study on the short-lived fish Nothobranchius furzeri (maximum recorded captive lifespan of 13 weeks), fish fed a resveratrol supplement did not develop the age-related neurofibrillary degeneration at nine weeks of age that developed in untreated fish [9]. Resveratrol was also shown to prevent an age-associated decline in learning in $N$. furzeri [9]. In male mice (Mus musculus), older individuals given resveratrol had better balance and motor coordination than untreated individuals of the same age [10].

However, there is little evidence that resveratrol can enhance cognition in healthy individuals in the absence of pathological or age-related cognitive decline. Recent studies on healthy mice and fish only demonstrated a significant difference in cognition between resveratrol-treated and untreated individuals once the untreated individuals had lived more than half their expected lifespan $[9,10]$. These results indicate that resveratrol provided some protection to the brain from the effects of aging, without measurably improving cognition in the short term. 
Resveratrol supplements are marketed as having the potential to improve cognitive function. Therefore, further studies are warranted to determine whether such supplements improve cognitive function in the absence of significant pathology. We investigated the effects of resveratrol on cognitive function in young, healthy, recently gonadectomised (neutered) dogs, as assessed by their performance in a radial arm maze. For comparison, we also investigated effects of gonadectomy on cognition, given evidence that gonadectomy can reduce cognitive function [11-14]. Dogs were chosen as the test subjects both because resveratrol supplements are being promoted for use in dogs as well as humans, and because dogs have been used as a model for the human brain in studies of aging [15-17].

\section{MATERIALS AND METHODOLOGY}

\subsection{Ethics Statement}

All procedures were approved by the University of Queensland Animal Ethics Committee and by the Iams Company's Institutional Care and Use Committee. Dogs were pair-housed in kennels with runs during the day and housed individually in kennels at night, and were fed commercial dog food at night. Each dog spent 10 to 20 minutes per day in a supervised play group inside a grass exercise yard (approximately 50 metres by 25 metres) that contained objects for the dogs to climb on. Groups of between two and six dogs were in the yard at a time. Whilst in the yard, dogs were not on leads and were able to interact with the dog handlers and the other dogs in the group. The dogs were also given toys (for example, plastic balls, rubber balls, treat balls, rope chews and rubber bones) and could play in a swimming pool. The dogs each received 10 minutes of clicker-based obedience training daily, plus they were routinely given commands when being handled (for example, they were asked to sit at gates and doors before going through). Dogs were re-homed after the completion of the study.

\subsection{Study Overview, Subjects and Treatments}

A prospective cohort study was used to compare the performance in radial arm maze trials between untreated nongonadectomised (intact) dogs, resveratrol-treated gonadectomised dogs and placebo-treated gonadectomised dogs. Dogs used in this study were 27 intact, mixed breed dogs involved in a nutrition trial investigating the effect of dietary supplements on weight gain. Dogs were sourced from council pounds and were selected for the nutritional trial based on bodyweight (12 to 30 kilograms), physical examination (clinically healthy), haematology, serum biochemistry, urinalysis and faecal examination. Only dogs with a suitable temperament for re-homing were included in the trial. Age could not be accurately determined but all dogs were estimated to be young adults over seven months of age, ranging from seven to 27 months. The median weight was 17.1 kilograms. Dogs were gonadectomised over a period of two weeks.

The 27 dogs were matched based on gender, bodyweight and body condition score. Each dog was assigned a supplement (resveratrol, from Japanese knotweed, 60mg/day) or a placebo (microcrystalline cellulose). Treatments were assigned using computer-generated random numbers. Each dog was started on the supplement or placebo two weeks after it was gonadectomised. These were given once daily at feeding time in microcrystalline cellulose capsules. Dogs were fed a dry extruded canine maintenance diet, and were fed in excess of maintenance energy requirements after gonadectomy.

\subsection{Testing Apparatus}

An eight-arm radial maze was used to test the spatial and working memory of the study dogs (Figs. 1 and 2). The maze was comprised of an aluminium frame covered with opaque vinyl, on a plywood base covered with vinyl. The maze con-

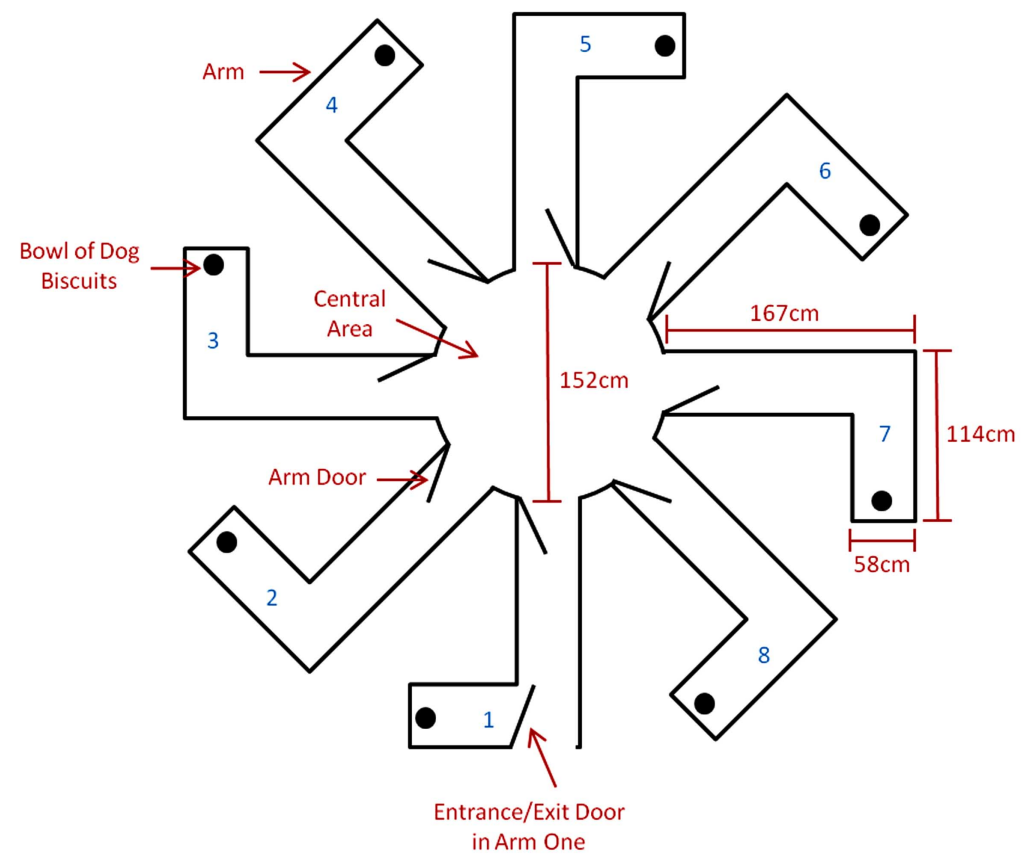

Fig. (1). Diagram, as viewed from above, of the eight-arm radial maze used to test the cognitive function of the dogs. 


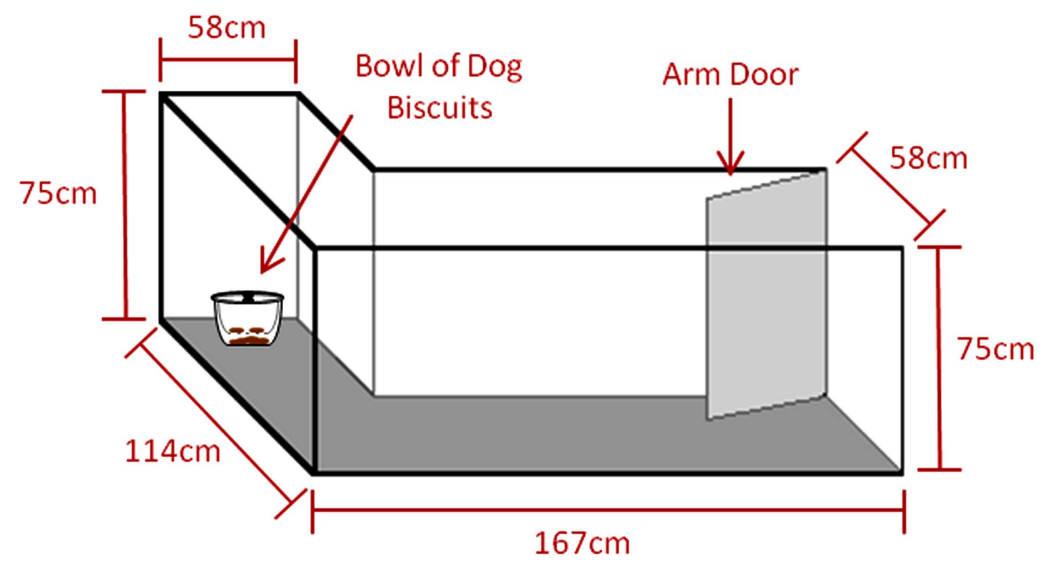

Fig. (2). Diagram, as viewed from the side, of one of the arms of the eight-arm radial maze used to test the cognitive function of the dogs.

sisted of eight, equally spaced, L-shaped arms that radiated outwards from a central circular area. Hinged doors were placed at the junction of each arm with the central area. These doors allowed each arm to be closed off independently. Arms were numbered one to eight (Fig. 1). The dogs entered and exited the maze through a door in arm number one. We chose the radial arm maze as the testing apparatus for our study as it is commonly used to test spatial and working memory in other species, and therefore comparisons with other species could be more easily made. It has been shown to be effective in this regard, particularly when measures that prevent the use of a physical strategy to complete the challenge are implemented [18, 19] (as was the case in phases two and three of our study).

Each arm was baited with commercial dog biscuits in double-layered microwave bowls (Fig. 3). To create a constant food odour at the end of each arm, five biscuits were placed in the bottom of each bowl and these were replaced daily. The top layer of each bowl was perforated to allow the smell of the biscuits in the bottom to escape and was clipped to the bottom layer to prevent the dogs accessing the bottom biscuits. Two more biscuits were placed in the top layer of the bowl as a food reward. The type of dog biscuit used was changed halfway through the study because the dogs appeared to be becoming less motivated by the original type of biscuit. Other odour clues in the maze were disguised by smearing and neutralising the odours using a mop. The maze was mopped before and after each dog's trial as well as between the forced and free choice sections of the trials in phases two and three. The dogs were restrained using a five metre extendable leash that was released during the trials. A stopwatch was used to measure the duration of each trial as well as the delay between the forced and free choice sections during which mopping took place.

\subsection{Testing Protocol}

Trials were carried out six mornings a week. A trial consisted of eight choices made by a dog. The dogs entered each maze arm in order to retrieve food rewards at the end of the arm. A choice was considered correct when a dog entered an arm it had not previously entered during that trial. An incorrect choice was made when a dog re-entered an arm it had previously entered during that trial. The dog had to move at least two-thirds of the way down an arm for it to be considered 'entered'. A marker was placed two-thirds of the way down each arm to ensure consistency. Typically, once a dog had moved two-thirds of the way down an arm, it continued on to the end of the arm and ate the food reward located there.

One person handled the dogs and stood in the centre of the maze during the trials. This person stood behind the dog and did not move during its trials, to avoid influencing the dog's choices. Another person stood in a set position outside the maze and recorded the dog's movements, the duration of the trials and the delay between the forced and free choice sections. The person recording the data only entered the maze to mop the floor and to open and close the doors. The data recorder stood at ground level as the entire maze could be easily viewed from that position. In order to reduce dis-

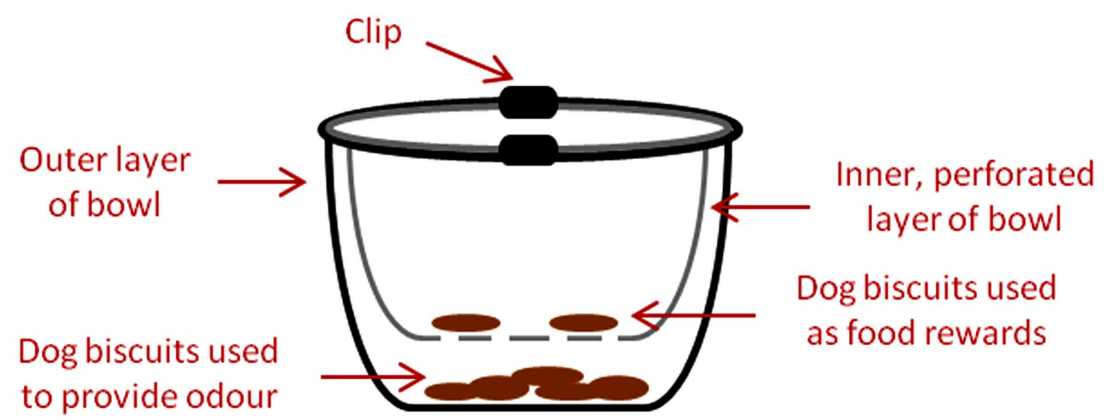

Fig. (3). Diagram of the double-layered microwave bowl used to hold dog biscuits at the end of the arms in the eight-arm radial maze involved in the testing of cognitive function of the dogs. 
tractions to the dogs, no other people were present during the trials.

The dogs were each given approximately 10 practice trials to familiarise them with the maze before testing started. During these practice trials, baited bowls were initially placed halfway along the arms, within the dogs' sight, to encourage them to enter the arms. Bowls were then moved during the course of the practice trials until the dogs would willingly move around the maze with the bowls at the ends of the arms. These practice trials were continued until the dogs found all the food rewards. Testing started approximately a month after the practice trials were held. The trials for the test sample of untreated intact dogs occurred several months before they were gonadectomised. The gonadectomised dogs were started on either the placebo or resveratrol two weeks after gonadectomy and their maze trials occurred two to four weeks after the treatments were initiated. The dogs received the treatments for 16 weeks in total. Testing was divided into three phases.

\subsubsection{Phase One: Assessment of Memory in a Free Choice Paradigm}

Phase one compared the performances of untreated intact dogs, resveratrol-treated gonadectomised dogs, and placebotreated gonadectomised dogs. Phase one was used to study the length of time it took for the dogs to learn the task, the development of choice patterns and the nature of errors made.

Twenty-seven dogs participated in phase one. Sixteen of these were tested before gonadectomy as untreated intact dogs. The other 11 dogs (six resveratrol-treated and five placebo-treated) were included after they had been gonadectomised. In phase one, the performances of untreated intact dogs $(n=16)$, resveratrol-treated gonadectomised dogs $(n=$ 6 ) and placebo-treated gonadectomised dogs $(n=5)$ were compared when the dogs were allowed to choose all the maze arms they entered. No dog was enrolled in more than one group.

At the start of each trial, the doors to all arms were opened. The dog was led into the centre of the maze and made to sit. The trial began when the extendable lead was released. The dog was allowed to explore the maze until it had found all food rewards or until 20 minutes had passed, but only the first eight arms entered in each trial counted towards the dog's score for that trial. There was provision for a dog to be given more time or allowed to repeat a trial if it had entered the maze arms less than eight times by the end of 20 minutes. Sequential arm choices were recorded. The time between consecutive trials for each dog was generally between one and six days. The trials were repeated until, over four consecutive trials, the dogs achieved an average of at least 7.2 correct choices out of their first eight choices in each trial, except for two dogs that had consistently low scores. Their testing was stopped once all other dogs had reached the success criterion. The numbers of trials required to reach the success criterion were compared between the three groups.

\subsubsection{Phase Two: Assessment of Memory in a Paradigm Involving Four Forced Choices and Four Free Choices}

Once the dogs reached the success criterion in phase one, they participated in phase two. The purpose of phase two was to force the dogs to use memory rather than a physical strategy to complete the task. Phase one could be completed by developing a physical strategy such as always choosing the arm to the left of the one previously entered. However, in phase two, the first four arms that the dogs entered were chosen for them using a system of random assignment without replication. Therefore, to successfully complete the tasks in phase two, the dogs had to remember which arms they had previously entered because a physical strategy would no longer be sufficient. The time between a dog finishing phase one and starting phase two depended on how long it took the dog to complete phase one and the availability of the researchers, with the median time being 19 days. Phase two compared the performances of untreated intact dogs $(n=15)$, resveratrol-treated gonadectomised dogs $(n=6)$ and placebo-treated gonadectomised dogs $(n=5)$. No dog was enrolled in more than one group. Two dogs failed to achieve the success criterion in phase one and one of these dogs was used in phase two. This dog was tested as an untreated intact dog in phase one but was tested in phase two as a placebotreated gonadectomised dog. The dog excluded from phase two was a placebo-treated gonadectomised dog in phase one. Dogs entered four predetermined arms one at a time (which constituted the forced choice section) and were then allowed to make four free choices from all eight arms.

At the start of each trial, the dog was led into the centre of the maze and made to sit. The doors to all arms were closed and one door was then opened. The dog was allowed to enter that arm and consume the food reward at the end of it. The dog was then made to sit in the centre of the maze and the door was closed. This was repeated with three other arms and constituted the forced choice section of the trial. These four arms and the order they were opened were randomly assigned before the trials using computer-generated random numbers.

Once the dog had returned from the fourth arm and was sitting in the centre of the maze, the area directly around the dog as well as part of each arm were mopped. In each arm, only the third closest to the dog was mopped. The doors to all of the arms were then opened. The extendable lead was released and the dog was allowed to enter the maze arms four times. The trial ended when the dog had made four free choices from all eight arms. Sequential arm choices and the delay between the forced and free choice sections were recorded for each trial. The delay varied between 25 and 45 seconds for individual trials but the mean length of the delay and standard deviation were similar for the three groups. The mean \pm standard deviation was $36.27 \pm 4.60$ seconds for the untreated intact dogs, $35.64 \pm 3.42$ seconds for the placebotreated gonadectomised dogs and $35.07 \pm 3.64$ for the resveratrol-treated gonadectomised dogs.

The time between consecutive trials for each dog was generally between one and six days. The trials were repeated until, over four consecutive trials, the dogs achieved an average of at least 3.2 correct choices out of the first four free choices in each trial, except for one dog that had consistently 
low scores. Its testing was stopped once all other dogs had reached the success criterion. The numbers of trials taken to reach the success criterion were compared between the three groups.

\subsubsection{Phase Three: Assessment of Memory in a Paradigm Involving Seven Forced Choices and One Free Choice}

In phase three, a subset of dogs previously tested as untreated intact dogs in phases one and two were tested as placebo-treated or resveratrol-treated gonadectomised dogs. Dogs entered seven predetermined arms one at a time (which constituted the forced choice section) and were then given the free choice between a previously opened arm and the arm that had not been previously opened. The first seven arms were systematically chosen for them, to study serial position effects (reported in a separate study ${ }^{1}$ ).

Phase three ran concurrently with the trials of the gonadectomised dogs in phase two. Twelve dogs tested as untreated intact dogs in phases one and two were tested as resveratrol-treated and placebo-treated gonadectomised dogs in phase three. In phase three, the performances of resveratroltreated gonadectomised dogs $(n=6)$ and placebo-treated gonadectomised dogs $(n=6)$ were compared. No dog was enrolled in more than one group.

At the start of each trial, the dog was led to the centre of the maze and made to sit. All the arm doors were closed and one door was then opened. The dog was permitted to enter that arm and consume the food reward at the end of it. The dog was then made to sit in the centre of the maze and the door was closed. This was repeated with six other arms and constituted the forced choice section of the trial. The arms used in the forced choice section and the sequence in which they were opened were randomly assigned before the trials using computer-generated random numbers.

Once the seven arms of the forced choice section had been entered, the dog was made to sit in the centre of the maze. After the floors were mopped, two doors were then simultaneously opened: (1) the door to one of the arms that the dog had previously entered in either serial position one, three, five or seven (serving as a distracter arm that had been randomly chosen using computer-generated random numbers); and (2) the door of the arm that the dog had not entered in the forced choice section of the trial. The area directly around the dog as well as part of these two arms had been mopped before the doors were opened. Within each arm, only the third closest to the dog was mopped. Once the doors were opened, the lead was released and the dog was allowed to enter one of the arms. The arm choices and the delay between the forced and free choice sections were recorded for each trial. The delay varied from 11 to 15 seconds. There were one to five days between consecutive trials for each dog, except for one period where no trials were performed for approximately three weeks. The number of trials performed per dog varied between 16 and 22 (mean 19.0). The percentages of trials where the dog chose the correct

\footnotetext{
${ }^{1}$ Craig M, Rand J, Shyan-Norwalt M et al. Domestic dogs demonstrate a primacy serial position effect using the radial arm maze. In press.
}

arm (that is, the previously un-entered arm) of the two offered were compared between the two groups.

\subsection{Statistical Analyses}

For phases one and two, the number of trials taken to reach the success criterion was analysed by comparing Kaplan-Meier survival functions in pair-wise comparisons between groups (untreated intact, placebo-treated gonadectomised and resveratrol-treated gonadectomised), using logrank tests. This approach allowed dogs that did not reach the success criterion to still contribute to the analyses; these dogs were right-censored at the number of their last trial. For phase three, the binary outcome variable (correct or incorrect arm chosen) was modelled using generalised estimating equations (GEE) with trial fitted within dog, with binomial error distributions and logit links, and with serial position of the incorrect door (the sequence number when that door was previously opened either first, third, fifth or seventh of the seven doors) and treatment (resveratrol or placebo) fitted as fixed effects. This approach was used to account for correlations (or lack of statistical independence) of repeated trials in the same dog. Such correlations seemed plausible as some dogs may have been correct consistently more than other dogs. Robust standard errors were used. Three models were fitted to analyse the phase three data. In the first model, an autoregressive correlation structure was fitted. This assumed that within each dog, the correlation between results for any pair of trials decreased with increasing time or (more specifically) with increasing numbers of trials between the pair of trials. An exchangeable correlation structure was fitted in the second model. This assumed the same correlation within each dog between any pair of trials. In the third model, trial number was fitted as a continuous variable with an exchangeable correlation structure; this accounted for any systematic linear change in log odds of dogs choosing the correct door with additional trials. All three models took into account the effects of the serial position of the distracter arm and therefore controlled for any confounding of primacy/recency on effects of treatment. The estimated effects of treatment (resveratrol or placebo) from the three models were very similar and so only the results of the first model are reported. Statistical analyses were performed using Stata 10.1 ® (StataCorp, College Station, Texas, USA).

\section{RESULTS}

\subsection{Effect of Gonadectomy on Memory}

\subsubsection{Phase One: Assessment of Memory in a Free Choice Paradigm}

Phase one assessed the effect of gonadectomy on the performance of dogs in an eight-arm radial maze when the dogs were allowed to choose all the arms they entered. Percentiles and medians of the number of trials taken to reach the success criterion in phase one (an average of at least 7.2 correct choices out of the first eight choices in each trial, over four consecutive trials) are summarised for each group in Table $\mathbf{1 .}$ The numbers of trials taken to reach the success criterion were not significantly different $(p=0.81)$ between untreated intact dogs $(n=16)$ and placebo-treated gonadectomised dogs $(n=5)$. One of the untreated intact dogs and one of the 
placebo-treated gonadectomised dogs failed to reach the success criterion.

\subsubsection{Phase Two: Assessment of Memory in a Paradigm Involving Four Forced Choices and Four Free Choices}

Phase two assessed the effect of gonadectomy on memory when the dogs were permitted to enter four predetermined arms and were then allowed to make four free choices from all eight arms. Percentiles and medians of the number of trials taken to reach the success criterion in phase two (an average of at least 3.2 correct choices out of the first four free choices in each trial, over four consecutive trials) are summarised for each group in Table 2 . There was no significant difference $(p=0.53)$ between the numbers of trials taken to reach the success criterion by the untreated intact dogs $(n=15)$ and the placebo-treated gonadectomised dogs $(n=5)$. One of the placebo-treated gonadectomised dogs failed to reach the success criterion.

\subsection{Effect of Resveratrol on Memory}

\subsubsection{Phase One: Assessment of Memory in a Free Choice Paradigm}

In phase one, the effect of resveratrol on the performance of dogs in an eight-arm radial maze was assessed when the dogs were allowed to choose all the arms they entered. Percentiles and medians of the number of trials taken to reach the success criterion in phase one (an average of at least 7.2 correct choices out of the first eight choices in each trial, over four consecutive trials) are summarised for each group in Table $\mathbf{1}$. There was no significant difference $(\mathrm{p}=0.52)$ in the numbers of trials taken to reach the success criterion between the placebo-treated gonadectomised dogs $(n=5)$ and the resveratrol-treated gonadectomised dogs $(n=6)$. One of the placebo-treated gonadectomised dogs failed to reach the success criterion.

\subsubsection{Phase Two: Assessment of Memory in a Paradigm Involving Four Forced Choices and Four Free Choices}

In phase two, the effect of resveratrol on the performance of dogs in an eight-arm radial maze was assessed when the dogs were permitted to enter four predetermined arms and were then allowed to make four free choices from all eight arms. Percentiles and medians of the number of trials taken to reach the success criterion in phase two (an average of at least 3.2 correct choices out of the first four free choices in each trial, over four consecutive trials) are summarised for each group in Table 2 . No significant difference $(p=0.15)$ was observed between the placebo-treated gonadectomised dogs $(n=5)$ and the resveratrol-treated gonadectomised dogs $(n=6)$ in the number of trials taken to reach the success criterion. One of the placebo-treated gonadectomised dogs failed to reach the success criterion.

\subsubsection{Phase Three: Assessment of Memory in a Paradigm Involving Seven Forced Choices and One Free Choice}

Phase three assessed the effect of resveratrol on the performance of dogs in an eight-arm radial maze when the dogs had to choose between an un-entered arm and an arm previously entered in serial position one, three, five or seven. The percentages of trials in which the placebo-treated gonadectomised dogs $(n=6)$ chose the correct arm $(99 / 118$ or

Table 1. Median and Upper and Lower Quartile Distribution for the Numbers of Trials in an Eight-arm Radial Maze that were Required for Dogs to Reach the Success Criterion (an Average of at Least 7.2 Correct Choices Out of the First Eight Choices in Each Trial, Over Four Consecutive Trials) in Phase One, where Dogs were Allowed to Choose from All Eight Arms

\begin{tabular}{|c|c|c|c|}
\hline Treatment group & 25th percentile & Median & 75th percentile \\
\hline Untreated intact dogs $(n=16)$ & 8.0 & 9.0 & 14.5 \\
\hline Resveratrol-treated gonadectomised dogs $(n=6)$ & 7.8 & 11.5 & 13.8 \\
\hline
\end{tabular}

Table 2. Median and Upper and Lower Quartile Distribution for the Numbers of Trials in an Eight-arm Radial Maze that were Required for Dogs to Reach the Success Criterion (an Average of at Least 3.2 Correct Choices Out of the First Four Free Choices in Each Trial, Over Four Consecutive Trials) in Phase Two, where Dogs were Permitted to Enter Four Predetermined Arms and were then Allowed to Make Four Free Choices from All Eight Arms

\begin{tabular}{|l|c|c|c|}
\hline \multicolumn{1}{|c|}{ Treatment group } & 25th percentile & Median & 75th percentile \\
\hline \hline Untreated intact dogs $(n=15)$ & 4.0 & $\mathbf{6 . 0}$ & 10.0 \\
\hline Placebo-treated gonadectomised dogs $(n=5)$ & 8.0 & $\mathbf{9 . 0}$ & 9.0 \\
\hline Resveratrol-treated gonadectomised dogs $(n=6)$ & 6.3 & $\mathbf{7 . 5}$ & 8.0 \\
\hline All dogs $(n=26)$ & 5.3 & $\mathbf{7 . 5}$ & 9.0 \\
\hline
\end{tabular}


83.9\%) and the resveratrol-treated gonadectomised dogs ( $n=$ 6 ) chose the correct arm (92/110 or $83.6 \%)$ did not differ significantly $(\mathrm{p}=0.98$; odds ratio $=0.99 ; 95 \%$ confidence interval 0.48 to 2.03 ).

\section{DISCUSSION}

Since there is evidence to suggest that gonadectomy can reduce cognitive function [11-14], we investigated whether the performance of gonadectomised dogs in a radial arm maze would be impaired compared to that of intact dogs. However, we found no significant difference in performance between gonadectomised dogs and intact dogs. This result was not completely unexpected as, in a study using rats, a significant difference in cognitive function between shamoperated rats and gonadectomised rats was only seen seven months after gonadectomy [20], which is longer than the time between gonadectomy and the start of testing in our study (which was four to six weeks).

We also assessed effects of resveratrol on cognitive function in gonadectomised dogs. Findings from several studies have demonstrated that performance of dogs in cognitive tests (including those that examine spatial memory) can be improved by various substances (including antioxidants, mitochondrial cofactors and a pharmaceutical with alpha-1 adrenergic agonist action) and by behavioural enrichment [21-25]. Oral administration of resveratrol has been used with success in previous studies of cognitive function in animals $[3,5,9,10]$. There are very few studies on the bioavailability of resveratrol in dogs. However, in two studies examining the toxicity of resveratrol in dogs, doses of 200 to $1200 \mathrm{mg} / \mathrm{kg}$ administered orally in capsules resulted in detectable levels of resveratrol and its metabolites in plasma [26, 27]. A biological effect on weight gain, body condition, body fat and energy consumption was evident in our dogs at $60 \mathrm{mg} / \mathrm{dog}$, indicating that resveratrol was bioavailable at this lower dose in dogs (unpublished data ${ }^{2}$ ). Furthermore, a study in humans demonstrated an effect of resveratrol at a dose of approximately $1.5 \mathrm{mg} / \mathrm{kg}$ [28], which is lower than the dose used in our study (2 to $5 \mathrm{mg} / \mathrm{kg}$ ). Finally, a resveratrol supplement is available for dogs at a recommended dose of $5 \mathrm{mg}$ per $13.6 \mathrm{~kg}$ body weight $\left(0.37 \mathrm{mg} / \mathrm{kg}^{3}\right)$. Therefore, we sought to investigate whether short-term oral treatment with resveratrol would improve the performance of young, healthy, recently gonadectomised dogs in an eight-arm radial maze. Based on our results, we conclude that short-term resveratrol treatment at $60 \mathrm{mg} / \mathrm{dog}$ does not improve the aspects of cognitive function assessed by these radial arm maze trials in young, healthy dogs managed in the same way as dogs in our study.

\footnotetext{
${ }^{2}$ Rae LS, Rand J, Morton J, et al. Dietary supplementation with resveratrol results in greater body weight gain after gonadectomy than supplementation with L-carnitine in healthy young adult dogs. J Vet Intern Med 2006 MayJun; 20: 793.

Rae LS, Rand J, Morton J, et al. Dietary supplementation of dogs with resveratrol after neutering results in a greater increase in body fat percentage but not substantially increased energetic requirements for tissue gain compared to dogs supplemented with L-carnitine. J Vet Intern Med 2007 MayJun; 21: 657.

${ }^{3}$ Resvantage Canine ${ }^{\circledR}$, Advantage Biosciences, 1580 Monrovia Ave, Newport Beach, California, USA.
}

One possible explanation for the absence of an observed effect of resveratrol in our study is that resveratrol acts by minimising deterioration in cognitive function, not by enhancing cognitive function. Many studies have indicated that resveratrol can minimise the deleterious effect on cognition of aging and physical and chemical damage to the brain [1-7, $9,10]$. Furthermore, resveratrol has been shown to prevent oxidative damage [8] and to clear the beta-amyloid peptides that are associated with Alzheimer's disease [29, 30]. However, there is no evidence that resveratrol enhances cognition in the absence of pathology (as is the situation in young, healthy individuals). One study in young rats showed that resveratrol did not affect the levels of several cerebral metabolites or improve the performance in maze tasks in normal individuals [5]. However, resveratrol did minimise the adverse effects of the neurotoxin colchicine on these parameters. If resveratrol can only protect the brain against damage (as opposed to being able to enhance its function), resveratrol would not be expected to produce a measurable effect on cognition in young, healthy individuals, such as the dogs in our study.

Another possible explanation for the lack of an observed effect of resveratrol in our study was the short duration of treatment. The dogs received resveratrol for only two to four weeks before testing began and the total duration of treatment was only four months. In previous studies where resveratrol was shown to have an effect on cognition, the subjects were given resveratrol for at least one third of their lifespan $[9,10]$. Short periods of resveratrol treatment in rats, such as a single dose [2] or 21 days of treatment [4], have produced positive effects on cognition, but the animals used in those studies had been subjected to neurological trauma or treatment with a neurotoxic substance. The failure to observe an effect after short-term treatment is a logical outcome if resveratrol's effect on cognitive function is mediated through protecting the brain against damage. In the previous studies where cognitive function was assessed in healthy animals, a significant effect of resveratrol was only seen when subjects were middle-aged or aged [9, 10]. In both Nothobranchius furzeri and mice, a significant difference in cognition between the resveratrol-treated individuals and untreated individuals was only seen once the untreated animals had lived more than half their expected lifespan [9, 10]. One logical explanation for the results of those studies is that resveratrol prevented the neurological pathology that is known to increase with age [31-34] but did not enhance cognition when the animals were young. The dogs in our study would not have aged much during the course of resveratrol treatment and so there would have been little brain pathology to prevent.

Our failure to observe an effect of resveratrol may also have been due to the effects of our procedures on the dogs' behavioural responses. Environmental/behavioural enrichment and extensive handling may interfere with the extent of any improvement in spatial tasks imparted by oestrogen [3537], and the dogs in our study received both extensive handling and environmental/behavioural enrichment. It is reasonable to suggest that these factors may also influence the extent of any positive effects of resveratrol on cognition as resveratrol has oestrogen-like effects [38]. Studies comparing mice exposed to enriched environments (provided by 
cage mates, toys and running wheels) and non-enriched environments have shown that, in certain tests of cognitive function, oestrogen appeared to only improve performance in non-enriched mice and not in enriched mice [35, 36]. In some of the tests, mice that received both oestrogen and enrichment performed worse than mice that received either oestrogen or enrichment [36]. In other tests, oestrogen failed to further enhance the performance of the enriched mice beyond the improvement caused by environmental enrichment; the enriched, oestrogen-treated mice and the enriched, placebo-treated mice did not differ significantly in performance but did perform significantly better than the non-enriched, placebo-treated mice [35]. In contrast, the non-enriched, oestrogen-treated mice performed significantly better than the non-enriched, placebo-treated mice in the same tests [35]. This pattern was also apparent when rats were exposed to increased handling [37]. In radial arm maze trials, oestradioltreated rats that received standard handling and placebotreated rats and oestradiol-treated rats that received increased handling all performed at a similar level that was significantly better than placebo-treated rats that received standard handling [37]. Thus, the oestradiol failed to enhance performance beyond the improvement caused by the increased handling [37]. This seems to indicate that a ceiling effect can occur and that, if cognitive performance is significantly improved by environmental enrichment or increased handling, oestrogen may not be able to further improve performance. The dogs in our study received environmental/behavioural enrichment through pair-housing, daily training, toys and a group exercise yard. Thus, the enrichment they received was similar to that given to the mice in the studies on oestrogen and enrichment. It was also very similar to the enrichment provided to the dogs in a study that showed that behavioural/environmental enrichment (provided by kennel mates, increased exercise, toys and a series of cognitive tests) improved the performance of aged dogs in black/white discrimination and reversal tasks [24]. Therefore, a ceiling effect may have also occurred in our study. The environmental enrichment that the dogs received may have improved their performance over what they would have otherwise achieved and the resveratrol supplementation may not have been able to further enhance their performance beyond that improvement.

Our failure to observe an effect of resveratrol may also be because our maze did not test the aspects of cognitive function in dogs that are enhanced by resveratrol. Previous studies on the effect of resveratrol on cognition in healthy animals used a shuttlebox task which tests operant learning [9], and a rotorod which evaluates balance and coordination [10] as opposed to the maze trials in our study which examined aspects of spatial and working memory. Resveratrol has been shown to improve the performance of rats in maze trials but the rats used in those studies had been given a neurotoxic substance $[4,5]$.

Given our findings, we suggest that further studies investigate, the effects of resveratrol in aged dogs with signs of cognitive deficits, and also, whether long-term supplementation from the time of gonadectomy protects against agerelated cognitive decline in dogs. Research such as our study has important implications for both human and canine health as dogs are known to develop age-related cognitive dysfunc- tion similar to that seen in humans [39] and dogs have previously been used as a model for the human brain in studies of aging [15-17]. Therefore, developments in the understanding of canine brain aging and its treatment could be used to direct similar research in people. In addition, it has been shown that the hepatic metabolism of resveratrol in humans is more similar to that of dogs than that of rats and mice [40], which suggests that studies that use dogs to investigate the effects of resveratrol may be better models when it comes to extrapolating the results to humans than studies using rats or mice.

\section{CONCLUSION}

We conclude that short-term resveratrol treatment does not improve (and gonadectomy does not impair) the cognitive function assessed by these radial arm maze trials in young, healthy dogs receiving extensive handling and environmental/behavioural enrichment. Further research is required in this area to allow consumers to be more informed about the value of resveratrol supplements.

\section{CONFLICT OF INTEREST}

The authors declare that they have no conflict of interest. Funding was provided by The Iams Company, Dayton, Ohio. Dr Beth Flickinger, from the Iams Company, and Dr ShyanNorwalt, formerly from the Iams Company, were involved in the study design, data collection, analysis, decision to publish and preparation of the manuscript.

\section{ACKNOWLEDGEMENTS}

The authors wish to thank Linda Oliver and Nicole Richards from the University of Queensland for care of, retraining and re-homing the study dogs, and Deanne Wayne for assistance with data collection.

\section{CONFLICT OF INTEREST}

The author(s) confirm that this article content has no conflicts of interest.

\section{REFERENCES}

[1] Schmatz R, Mazzanti CM, Spanevello R, et al. Resveratrol prevents memory deficits and the increase in acetylcholinesterase activity in streptozotocin-induced diabetic rats. Eur J Pharmacol 2009; 610: 42-8.

[2] Sonmez U, Sonmez A, Erbil G, Tekmen I, Baykara B. Neuroprotective effects of resveratrol against traumatic brain injury in immature rats. Neurosci Lett 2007; 420: 133-7.

[3] Dong WP, Li NL, Gao DK, et al. Resveratrol attenuates ischemic brain damage in the delayed phase after stroke and induces messenger RNA and protein express for angiogenic factors. J Vasc Surg 2008; 48: 709-14.

[4] Sharma M, Gupta YK. Chronic treatment with trans resveratrol prevents intracerebroventricular streptozotocin induced cognitive impairment and oxidative stress in rats. Life Sci 2002; 71: 2489-98.

[5] Kumar A, Naidu PS, Seghal N, Padi SSV. Neuroprotective effects of resveratrol against intracerebroventricular colchicine-induced cognitive impairment and oxidative stress in rats. Pharmacology 2007; 79: 17-26.

[6] Kumar P, Padi SSV, Naidu PS, Kumar A. Effect of resveratral on 3-nitropropionic acid-induced biochemical and behavioural changes: possible neuroprotective mechanisms. Behav Pharmacol 2006; 17: 485-92. 
[7] Lu K, Ko M, Chen B, et al. Neuroprotective effects of resveratrol on MPTP-induced neuron loss mediated by free radical scavenging. J Agric Food Chem 2008; 56: 6910-3.

[8] Mokni M, Elkahoui S, Limam F, Amri M, Aouani E. Effect of resveratrol on antioxidant enzyme activities in the brain of healthy rat. Neurochem Res 2007; 32: 981-7.

[9] Valenzano DR, Terzibasi E, Genade T, et al. Resveratrol prolongs lifespan and retards the onset of age-related markers in a shortlived vertebrate. Curr Biol 2006; 16: 296-300.

[10] Pearson KJ, Baur JA, Lewis KN, et al. Resveratrol delays agerelated deterioration and mimics transcriptional aspects of dietary restriction without extending life span. Cell Metab 2008; 8: 157-68.

[11] Wallace M, Luine V, Arellanos A, Frankfurt M. Ovariectomized rats show decreased recognition memory and spine density in the hippocampus and prefrontal cortex. Brain Res 2006; 1126: 176-82.

[12] Gibbs RB, Johnson DA. Sex-specific effects of gonadectomy and hormone treatment on acquisition of a 12-arm radial maze task by Sprague Dawley rats. Endocrinology 2008; 149: 3176-83.

[13] Kritzer MF, McLaughlin PJ, Smirlis T, Robinson JK. Gonadectomy impairs T-maze acquisition in adult male rats. Horm Behav 2001; 39: 167-74.

[14] Hart BL. Effect of gonadectomy on subsequent development of age-related cognitive impairment in dogs. J Am Vet Med Assoc 2001; 219: 51-6.

[15] Head E. Combining an antioxidant-fortified diet with behavioral enrichment leads to cognitive improvement and reduced brain pathology in aging canines - Strategies for healthy aging. Ann NY Acad Sci 2007; 1114: 398-406.

[16] Araujo JA, Studzinski CM, Head E, Cotman CW, Milgram NW. Assessment of nutritional interventions for modification of ageassociated cognitive decline using a canine model of human aging. Age 2005; 27: 27-37.

[17] Studzinski CM, Araujo JA, Milgram NW. The canine model of human cognitive aging and dementia: Pharmacological validity of the model for assessment of human cognitive-enhancing drugs. Prog Neuropsychopharmacol Biol Psychiatry 2005; 29: 489-98.

[18] Olton DS, Collison C, Werz MA. Spatial memory and radial arm maze performance of rats. Learn Motiv 1977; 8: 289-314.

[19] Dubreuil D, Tixier C, Dutrieux G, Edeline JM. Does the radial arm maze necessarily test spatial memory? Neurobiol Learn Mem 2003; 79: 109-17.

[20] Markowska AL, Savonenko AV. Effectiveness of estrogen replacement in restoration of cognitive function after long-term estrogen withdrawal in aging rats. J Neurosci 2002; 22: 10985-95.

[21] Milgram NW, Araujo JA, Hagen TM, Treadwell BV, Ames BN. Acetyl-L-carnitine and alpha-lipoic acid supplementation of aged beagle dogs improves learning in two landmark discrimination tests. FASEB J 2007; 21: 3756-62.

[22] Milgram NW, Siwak CT, Gruet P, et al. Oral administration of adrafinil improves discrimination learning in aged beagle dogs. Pharmacol Biochem Behav 2000; 66: 301-5.

[23] Milgram NW, Head E, Zicker SC, et al. Long-term treatment with antioxidants and a program of behavioral enrichment reduces agedependent impairment in discrimination and reversal learning in beagle dogs. Exp Gerontol 2004; 39: 753-65.
[24] Milgram NW, Head E, Zicker SC, et al. Learning ability in aged beagle dogs is preserved by behavioral enrichment and dietary fortification: a two-year longitudinal study. Neurobiol Aging 2005; 26: 77-90.

[25] Nippak PMD, Mendelson J, Muggenburg B, Milgram NW. Enhanced spatial ability in aged dogs following dietary and behavioural enrichment. Neurobiol Learn Mem 2007; 87: 610-23.

[26] Crowell J, McCormick D, Cwik M, Kapetanovic I. Toxicokinetics of resveratrol in dogs. Toxicol Lett 2007; 172: S102.

[27] Johnson WD, Morrissey RL, Usborne AL, et al. Subchronic oral toxicity and cardiovascular safety pharmacology studies of resveratrol, a naturally occurring polyphenol with cancer preventive activity. Food Chem Toxicol 2011; 49: 3319-27.

[28] Timmers S, Konings E, Bilet L, et al. Calorie restriction-like effects of 30 days of resveratrol supplementation on energy metabolism and metabolic profile in obese humans. Cell Metab 2011; 14: 612-22.

[29] Marambaud P, Zhao HT, Davies P. Resveratrol promotes clearance of Alzheimer's disease amyloid-beta peptides. J Biol Chem 2005; 280: 37377-82.

[30] Karuppagounder SS, Pinto JT, Xu H, et al. Dietary supplementation with resveratrol reduces plaque pathology in a transgenic model of Alzheimer's disease. Neurochem Int 2009; 54: 111-8.

[31] Shigenaga MK, Hagen TM, Ames BN. Oxidative damage and mitochondrial decay in aging. Proc Natl Acad Sci U S A 1994; 91: 10771-8.

[32] Siwak-Tapp CT, Head E, Muggenburg BA, Milgram NW, Cotman CW. Region specific neuron loss in the aged canine hippocampus is reduced by enrichment. Neurobiol Aging 2008; 29: 39-50.

[33] Tapp PD, Siwak CT, Gao FQ, et al. Frontal lobe volume, function, and beta-amyloid pathology in a canine model of aging. J Neurosci 2004; 24: 8205-13.

[34] Head E, Liu J, Hagen TM, et al. Oxidative damage increases with age in a canine model of human brain aging. J Neurochem 2002; 82: $375-81$

[35] Gresack JE, Frick KM. Environmental enrichment reduces the mnemonic and neural benefits of estrogen. Neuroscience 2004; 128: 459-71.

[36] Gresack JE, Kerr KM, Frick KM. Short-term environmental enrichment decreases the mnemonic response to estrogen in young, but not aged, female mice. Brain Res 2007; 1160: 91-101.

[37] Bohacek J, Daniel JM. Increased daily handling of ovariectomized rats enhances performance on a radial-maze task and obscures effects of estradiol replacement. Horm Behav 2007; 52: 237-43.

[38] Bowers JL, Tyulmenkov VV, Jernigan SC, Klinge CM. Resveratrol acts as a mixed agonist/antagonist for estrogen receptors alpha and beta. Endocrinology 2000; 141: 3657-67.

[39] Cotman CW, Head E. The canine (dog) model of human aging and disease: dietary, environmental and immunotherapy approaches. J Alzheimers Dis 2008; 15: 685-707.

[40] Maier-Salamon A, Boehmdorfer M, Thalhammer T, Szekeres T, Jaeger W. Hepatic glucuronidation of resveratrol: interspecies comparison of enzyme kinetic profiles in human, mouse, rat, and dog. Drug Metab Pharmacokinet 2011; 26: 364-73. 